\title{
Gemini in RoboCup-2000
}

\author{
Masayuki Ohta \\ Tokyo Institute of Technology
}

\begin{abstract}
We implemented "Gemini" a client program for the SoccerServer. The objective of this program is testing a lot of learning methods on multi-agent environments. In the current implementation, Gemini can select the most effective strategy for an enemy, using reinforcement learning. Furthermore, we are trying to implement a meta-level learning, which turn each learning function on or off according to whether the learning succeed or not.
\end{abstract}

\section{Introduction}

Because it is hard to adjust behavior of each agent in multi-agent environments, machine learning techniques are necessary. We implemented "Gemini" a client program for the SoccerServer[3], to test some kinds of reinforcement learnings[1] on it. In particular, we are interested in learning cooperative behaviors under the following restrictions.

- Agent can get no information from the global viewpoint

- Sensory data includes some errors

- Agent can not communicate with each other

In such environment, reinforcement learning has been applied in many cases. However, because reinforcement learning progress to maximize the reward of the agent itself, when using reinforcement learning in this environment, there is a problem that reward of the whole team may not be maximized. Moreover, when we realize online learning, we can not avoid the possibility of unsuccessful learning. Then, some methods to reduce risks of online learning become necessary, because the risk is increased in such noisy environment.

In this paper, we show the outline of Gemini's architecture and the learning system to solve these problems.

\section{Architecture}

Gemini mainly consists of the following five modules as Figure 1: Network Interface, Sensor, World Model, Controller and Learning Module. Learning Module includes some learning functions, each of which can have different kind of learning method. Gemini execute the followings in every simulation cycle. This loop will be repeated until the end of the simulation. 


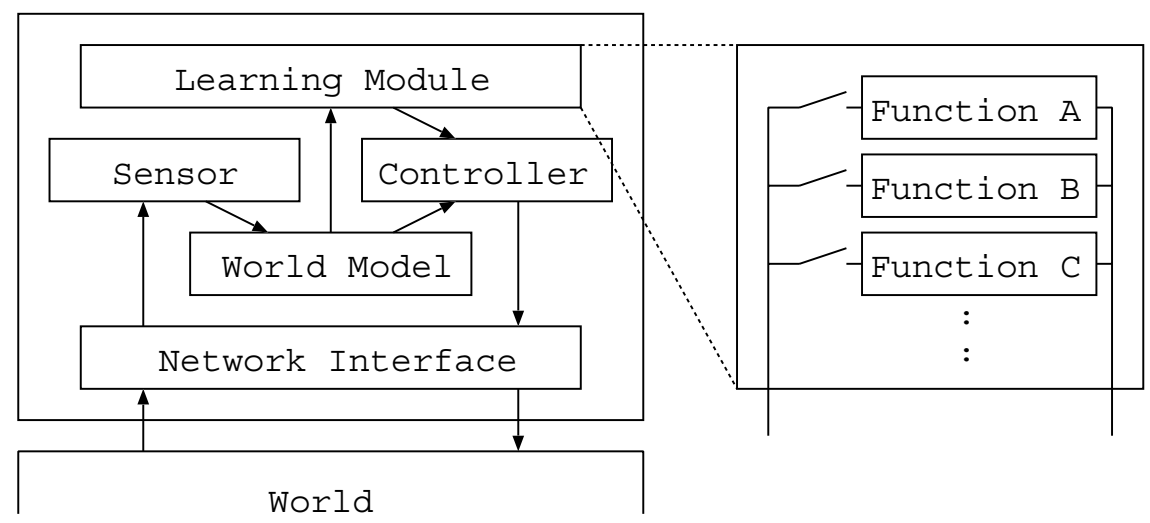

Fig. 1. Structure of Gemini

1. Sensor receives perception information through Network Interface, and put it into World Model.

2. Learning Module executes some learning method, and make some changes to Controller.

3. Controller decides the next action and send control commands to the SoccerServer through Network Interface.

4. World Model estimates the next object's positions and velocities.

The action rules in the Controller are written in "if-then rules". Considering the World Model, the Controller decides the next action based on this rules. In the "learning and change strategies" phase, some learning functions calculate new variables for the if-then rules in the Controller. Then, Gemini can change it's action rule.

At the beginning, all the players (except for the goal keeper) behave just the same. Start with different position, each player learn their best behavior respectively. This makes the difference of actions among each part.

\section{Learning}

Gemini realizes both online learning and offline learning with the same mechanism. To reduce the risk of online learning, it also have a meta-level learning mechanism, which select only effective learning methods.

\subsection{Select the Best Strategy}

Gemini get to select the most effective strategy against the opponent with reinforcement learning. There are some learning functions in the learning module, 
and each learning function progresses individually. Variables for Controller are calculated by these functions, and they have influence on behaviors such as "base position", "from which side to attack" and "whether do centering or shoot directly". A learning function has a neural network, and learns the best strategy by improving it. We are using back propagation to refine the neural network, and give a reward of reinforcement learning as an expected value of the output.

For example, the learning function to decide "from which side to attack" acquires an expected reward of each policy as following.

- In advance, divide the field into several parts(cell). The division is not changed during the game.

- Neural network calculates the expected reward of each policy using "position of the ball" and "the number of the opponent in each cell" for the input.

- Select the policy whose expected reward is the highest, and make some changes into variables for target point. (To do exploration, there is a small possibility to select random policy, too.)

- Every five seconds, if the ball moves forward, expected reward for the policy is increased and refine the network with back propagation with the new expected reward.

In this kind of reinforcement learning, each agent evolves only for their own reward. To raise whole reward of the team without communication, we allow all agents to get reward and reinforce the policy at that time even if the successful is due to other agents[4].

This method can also be used for online learning. But exploration is sometimes very risky, and frequent changes of strategy causes bad influence to the learning of the teammates. Therefore, when we use this method for online learning, Gemini stores each reward while play on, and changes the strategy all at once when the play stops.

\subsection{Meta-Level Learning}

Gemini operates more than one learning mechanism simultaneously, but all the learning methods do not always go well. To use effective learning function selectively, Gemini has a meta-level learning function that turn on and off each learning.

In the recent implementation, we realize meta-level learning as follows. All the learning functions start with active state. During the simulation, the metalevel learning function records how much reward does each learning function get. If there are some learning function whose reward going down, meta-level learning function inactivate the learning function. Inactive learning functions become active by a random timing, but if the function is inactivated repeatedly the interval goes longer. 


\section{Summary}

In this paper, we describe the outline of Gemini for the RoboCup 2000. It can select the best strategy against opponents with online learning. To maximize the whole reward of the team in no communication environment, we adopt unique way to reward. Furthermore, because the online learning is so risky, the learning module has a mechanism to evaluate the effect of each learning function, and can use only effective learning selectively.

\section{References}

1. Kaelbling L. P., Littman M. L. and Moore A. W. "Reinforcement Learning: A Survey" Journal of Artificial Intelligence Research 4, pages 237-285 1996.

2. Kimura H., Yamamura M. and Kobayashi S. "Reinforcement Learning in Partially Observable Markov Decision Processes: A Stochastic Gradient Method" Journal of Japanese Society for Artificial Intelligence, Vol.11, No.5 papges 761-768 1996

3. Itsuki Noda, Hitoshi Matsubara, Kazuo Hiraki and Ian Frank. Soccer Server: A Tool for Research on Multiagent Systems. Applied Artificial Intelligence, Vol.12, pages 233-250, 1998.

4. M.Ohta and T.Ando "Cooperative Reward in Reinforcement Learning" Proc. of 3rd JSAI RoboMech Symposia pages 7-11 April 1998. 